\title{
Elements of Dynamic Economic Modeling: Presentation and Analysis
}

Leigh Tesfatsion

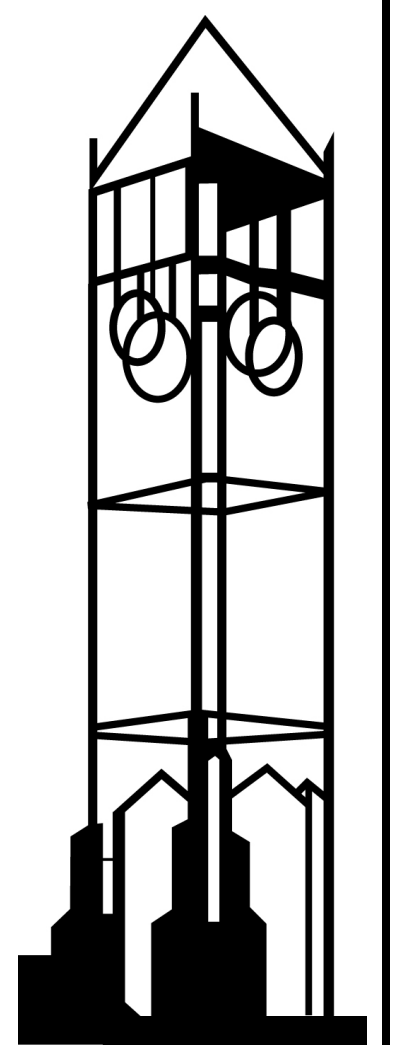

Working Paper No. 14001

February 2014

IOWA STATE UNIVERSITY

Department of Economics

Ames, lowa, 50011-1070

lowa State University does not discriminate on the basis of race, color, age, religion, national origin, sexual orientation, gender identity, genetic information, sex, marital status, disability, or status as a U.S. veteran. Inquiries can be directed to the Director of Equal Opportunity and Compliance, 3280 Beardshear Hall, (515) 294-7612. 
Last Updated: 18 February 2014

\title{
Elements of Dynamic Economic Modeling: Presentation and Analysis
}

\author{
Leigh Tesfatsion \\ Professor of Economics, Mathematics, and \\ Electrical \& Computer Engineering \\ Iowa State University, Ames, IA 50011-1070 \\ http://www.econ.iastate.edu/tesfatsi/
}

Abstract: The primary goal of these introductory notes is to promote the clear presentation and rigorous analysis of dynamic economic models, whether presented in equation or agent-based form. A secondary goal is to promote the use of state-space modeling with its respect for historical process, for cause leading to effect without top-down imposition of global constraints. If economic modelers truly wish to respect the rationality of decision-makers, they should have the courage of their convictions; they should not be doing for their modeled decision-makers what in reality these decision-makers must do for themselves.

\section{Overview}

These notes on dynamic economic modeling are designed for self-study by graduate students of economics. The focus is on general presentation and analysis principles for dynamic economic models expressible in state-space form.

A state-space model is a representation of a system for which, at each time $t$, future states of the system are expressible as possibly stochastic functions of the time- $t$ state, time$t$ actions, and time- $t$ realizations for stochastic elements, conditional on exogenous structural aspects such as postulated institutional arrangements, physical laws, and external probability distributions (e.g., for weather events). 
As will be clarified below, state-space models do not rule out forward planning and intertemporal optimization by modeled decision-makers. However, they do rule out ahistorical representations in which the actions of decision-makers are subject to intertemporal coordination restrictions requiring global solution methods, such as global fixed point determinations. They also rule out Delphic Oracle decision-makers with exact current knowledge of future state realizations.

The principles covered in these notes are illustrated by means of simple examples for continuous-time and discrete-time state-space models in equation form, and for discretetime state-space models in agent-based form. Extensive annotated pointers to additional materials on dynamic economic modeling, including specific forms of growth models (descriptive, optimal, overlapping-generations, dynamic stochastic general equilibrium, agent-based), expectation formation (adaptive, rational), and the constructive modeling of coordination processes for economic systems, can be found at the course website [1].

\section{General Presentation Considerations}

The basic requirement for the effective presentation in written form of an economic study conducted by means of a state-space model is to put yourself in the place of a potential reader.

The first thing a reader will want to see is a brief but clear summary statement of your study's purpose. The second thing the reader will want to see is a brief but clear discussion clarifying the extent to which your study has new aspects relative to what has been studied before, and why these new aspects are important for the achievement of your study's purpose. This could constitute your Section 1.

Once you secure the reader's interest with this introductory overview, your next task is to explain to the reader why you are choosing to support your study's purpose by means of your model. You should first provide the reader with a big-picture overview of your model that establishes its relationship to your study's purpose. This overview should consist of a concise but clear verbal discussion, perhaps with an accompanying flow diagram indicating in 
simplified form the flow of activities at successive points in time (continuous-time modeling) or over successive time periods (discrete-time modeling). The objective here is to convey in general terms how your model captures aspects of a dynamic economic system important for your study's purpose. This could constitute your Section 2.

Once the reader has a big-picture understanding of your model, you need to provide a more detailed explanation of the structure of the model. As will be clarified in subsequent sections, this explanation should include:

(i) a complete and consistent specification of simultaneous and dynamic relationships detailing what system events occur at each time $t$ (or during each time period $t$ ) and how the state of the system changes from one time point (or time period) $t$ to the next;

(ii) a listing of all variables and functions appearing in relationships (i), together with their intended economic meaning;

(iii) a classification for each variable and function in (ii) as either endogenous (model determined) or exogenous (given from outside the model);

(iv) a unit of measurement for each variable in (ii);

(v) a domain and range for each function in (ii);

(vi) admissibility restrictions on the exogenous variables and functions in (ii) needed to ensure the economic meaningfulness of the relationships (i), both individually and as a whole, as well as the economic meaningfulness of any resulting solution values for the endogenous variables.

The detailed aspects (i)-(vi) of your model's structure would typically be given in a separate Section 3.

You then need to explain carefully to the reader what type of analysis you intend to undertake with your model, in accordance with the purpose you have stated for your study. For example, do you intend to use the model to predict model outcomes under a particular 
empirically-determined specification of the exogenous variables and functions? In this case empirical input data will have to be given. Alternatively, do you intend to conduct sensitivity tests (analytical or computational) to determine model outcomes for a specified range of values for some subset of the exogenous variables? In this case you will need to explain to the reader the intended design for your sensitivity tests.

More precisely, to convey to the reader your sensitivity design, ${ }^{1}$ you will first need to explain carefully to the reader which of the exogenous variables appearing in your model are treatment factors, in the sense that they will be systematically varied during your sensitivity tests, and which are maintained factors in the sense that they will be maintained at fixed values throughout your sensitivity tests. You will then need to report to the reader the particular configurations of values you plan to test for your treatment factors, and the particular fixed values you plan to set for your maintained factors. If your model includes stochastic terms, you will also need to report the pseudo-random number seed values used in your sensitivity tests to generate multiple different runs for each tested treatment-factor configuration in order to analyze and control for stochastic effects. ${ }^{2}$

After the form of your analysis is fully explained to the reader, say in Section 4, the outcomes of your analysis would then typically be reported to the reader in a separate Section 5. The manner in which these outcomes are reported should be tailored to your study's purpose and form of analysis. For example, outcomes might be reported by means of verbal summaries, tables, charts, heat maps, phase diagrams, and/or various other types of figures.

The final section of your study should be a wrap-up section. Typically this section will start by summarizing the main accomplishments of your study as reported in previous sections. It will then identify interesting new questions or issues raised by the study that would be of interest to explore in subsequent studies.

\footnotetext{
${ }^{1}$ Sensitivity design is sometimes referred to as experimental design, given the formal similarity between sensitivity testing and laboratory experimentation. However, some researchers argue that the term "experiment" should be reserved for a sensitivity test conducted on a natural system, not on a theoretical construct. The use of "experiment" is therefore avoided in these notes.

${ }^{2}$ See [2] for a more extended discussion of sensitivity design for stochastic dynamic models.
} 


\section{Classification of Variables for State-Space Models}

A variable whose value is determined within a model is said to be endogenous (relative to that model). A variable whose value is determined outside of a model is said to be exogenous (relative to that model).

In dynamic econometric models, "predetermined variables" are defined to be exogenous plus lagged endogenous variables. However, for dynamic economic models in state-space form it is useful to distinguish between variables that are truly exogenous to the model and variables that are endogenous to the model but that have their values determined prior to other endogenous variables appearing within the model.

The following definitions clarify these endogenous variable distinctions. These definitions are applicable for both continuous-time and discrete-time state-space models.

A variable appearing in the time- $t$ equations for a state-space model is said to be time-t predetermined (relative to that model) if it is an endogenous variable whose value at time $t$ is determined by means of model equations for times $s<t$. A variable appearing in the time- $t$ equations for a state-space model is said to be time-t endogenous (relative to that model) if it is an endogenous variable whose value at time $t$ is determined by means of these time- $t$ equations.

The time- $t$ predetermined variables for a state-space model constitute the time- $t$ state variables for this model, and the vector of these time- $t$ state variables is referred to as the time-t state vector. As will be seen below, the state vector for a state-space model specified over times (or time periods) $t \geq 0$ needs to be exogenously given at time 0 since there are no model equations prior to this initial time. 


\section{Continuous-Time State-Space Modeling in Equation Form}

\subsection{Model Description}

A complete description for a continuous-time state-space model in equation form over time points $t \geq 0$ consists of two parts: a set of equations for each time $t$ describing postulated functional relationships among a collection of variables; and a classification of these variables and functions into time- $t$ endogenous, time- $t$ predetermined, and exogenous. An illustrative example is given below:

Continuous-time model equations for times $t \geq 0$ :

$$
\begin{aligned}
\text { Simultaneous: } \quad & =H(z(t), x(t), \alpha(t)) \\
\text { Dynamic: } & D x(t)=S(z(t), x(t), \alpha(t)) \\
\text { Integral: } & x(t)=\int_{0}^{t} D x(s) d s+x(0)
\end{aligned}
$$

\section{Variables and functions:}

$$
\begin{aligned}
& z(t)=\left(z_{1}(t), \ldots, z_{m}(t)\right) \in R^{m}, t \geq 0 \\
& D x(t)=\left(D x_{1}(t), \ldots, D x_{n}(t)\right) \in R^{n}, t \geq 0 \\
& x(t)=\left(x_{1}(t), \ldots, x_{n}(t)\right) \in R^{n}, t \geq 0 \\
& \alpha(t)=\left(\alpha_{1}(t), \ldots, \alpha_{k}(t)\right) \in R^{k}, t \geq 0 \\
& H: R^{m+n+k} \rightarrow R^{m} \\
& S: R^{m+n+k} \rightarrow R^{n}
\end{aligned}
$$

\section{Classification of variables and functions:}

Time- $t$ endogenous variables for times $t \geq 0: z(t), D x(t)$ 
Time-t predetermined (state) variables for times $t>0: x(t)$

Exogenous variables: $x(0), \alpha(t)$ for times $t \geq 0$

Exogenous functions: $H: R^{m+n+k} \rightarrow R^{m}$ and $S: R^{m+n+k} \rightarrow R^{n}$

Note that the above model contains $m+n$ equations (1) and (2) for the $m+n$ time$t$ endogenous variables $\left(z_{1}(t), \ldots, z_{m}(t)\right)$ and $\left(D x_{1}(t), \ldots, D x_{n}(t)\right)$, for each $t \geq 0$, and $n$ equations (3) for the $n$ time-t predetermined variables $\left(x_{1}(t), \ldots, x_{n}(t)\right)$ for each $t>0$. These variables thus constitute the set of all endogenous (model-determined) variables for the model as a whole.

We therefore could have used a simpler two-way classification of variables for this model, into endogenous and exogenous variables. However, for the analysis of state-space models it is often highly useful to use a slice-of-time approach in which the logical structure of the model is analyzed at each successive time $t$. The additional breakdown of endogenous variables into time- $t$ endogenous variables and time- $t$ predetermined variables then conveys important additional information concerning dynamic relationships among the endogenous variables. In particular, it shows which endogenous variables are determined prior to other endogenous variables.

As seen in [3], the classic continuous-time Solow-Swan descriptive growth model can be expressed in the above model form.

\subsection{Incorporation of Optimization Problems}

Dynamic economic models can (and typically do) include optimization problems for key types of decision-makers (firms, consumers, and/or government entities). These optimization problems are often constrained to ensure the existence of unique solutions (or are assumed to have unique solutions) for which first-order necessary conditions are also sufficient. In this case these optimization problems can be represented by their sets of binding first-order necessary conditions. 
For simple types of market models, these binding first-order necessary conditions could take the form of demand and supply functions derived by utility-maximizing consumers and profit-maximizing firms. For traditional Ramsey optimal growth models in calculusof-variations form, these conditions take the form of Euler-Lagrange equations constraining per-capita consumption and capital along an intertemporal solution path together with transversality conditions constraining the length of the planning horizon and the terminal per-capita capital stock. ${ }^{3}$

For more general optimization problems subject to inequality and/or equality constraints, these binding first-order necessary conditions might take the form of binding Karush-KuhnTucker conditions. They might also be expressed in terms of Hamiltonian conditions (in accordance with variants of Pontryagin's optimal control theory) or in terms of recursive relationships among successive dynamic programming value functions (in accordance with variants of Bellman's Principle of Optimality). ${ }^{4}$

These binding first-order necessary conditions can be incorporated among the equations of a state-space model as long as they are expressible in state-space form. More precisely, each decision-maker's objective function, feasible decision set, and decision selection method at each time $t$ must be expressible in terms of his information state at time $t$, where this information state constitutes part of the system state at time $t$. In particular, any future events represented in a decision-maker's current objective function must be expressed in terms of the decision-maker's anticipations regarding these future events, as determined by his current information state.

However, it is important to note that the set of binding necessary conditions for an optimizing decision-maker can change through time as anticipations are replaced by realizations if this decision-maker is allowed to re-optimize his intertemporal plans as time proceeds. This would result in state-dependent changes in the structure of the state-space model equa-

\footnotetext{
${ }^{3}$ Ref. [4] provides a detailed derivation and analysis of the Euler-Lagrange equations and transversality conditions for an illustrative Ramsey optimal growth model.

${ }^{4}$ See refs. [5] through [7] for excellent introductory discussions of these optimization tools for economic applications.
} 
tions over time. It could also result in time-inconsistency in the sense that the re-optimizing decision-makers deliberately choose to deviate from their earlier intertemporal plans as time proceeds. These issues are not addressed in these elementary notes. ${ }^{5}$

\subsection{Model Solutions}

Let a continuous-time state-space model in equation form be given, defined for $t \geq 0$. A solution for this model is a value for each endogenous variable determined as a function of exogenous variables and functions, where these values satisfy the model equations starting from the exogenously given initial state vector at time 0 .

Given that each time- $t$ predetermined variable $(t>0)$ is a time- $s$ endogenous variable for some earlier time $s$, an equivalent definition is as follows: A solution for this model is a value for each time- $t$ endogenous variable determined as a function of time- $t$ predetermined variables and/or exogenous variables and functions, where these values satisfy the model equations starting from the exogenously given initial state vector at time 0 .

In general, without further restrictions on model structure, there is no guarantee that a solution exists. ${ }^{6}$ Moreover, even if a solution exists, there is no guarantee it can be expressed in closed form, i.e., in the form of explicit functions mapping exogenous model elements into solution values for the endogenous variables. However, these issues will not be addressed in these elementary notes.

Suppose, then, that a closed-form solution exists for the illustrative continuous-time state-space model in Section 4.1 over the full time-range $t \geq 0$. In principle, this closed-form solution can be found as follows. First, for each time $t$, use equation (1) to solve for $z(t)$ as a function of $x(t)$ and $\alpha(t)$, say

$$
z(t)=g(x(t), \alpha(t)), \quad t \geq 0 .
$$

\footnotetext{
${ }^{5}$ An introductory discussion of these issues can be found in [8].

${ }^{6}$ The Cauchy-Peano Theorem demonstrates how difficult it is to find general conditions guaranteeing the existence of solutions for nonlinear systems of ordinary differential equations except locally, in an arbitrarily small neighborhood of the initial starting time. See Chapter 3.B in Takayama [5] for a discussion of existence and stability issues for such systems.
} 
Next, substitute (4) into equation (2), thus eliminating $\mathrm{z}(\mathrm{t})$, which then gives $\mathrm{Dx}(\mathrm{t})$ as a function of $x(t)$ and $\alpha(t)$ :

$$
D x(t)=S(g(x(t), \alpha(t)), x(t), \alpha(t)) \equiv f(x(t), \alpha(t)), \quad t \geq 0 .
$$

Given equations (4) and (5), plus the exogenous initial state vector $x(0)$, equations (3) permit the successive integral calculation of the solution values for the time- $t$ predetermined vectors $x(t), t>0$.

In summary, equations (3), (4), and (5) together permit the determination of a solution value for each endogenous variable as a function of exogenous model elements; hence, together they provide the desired closed-form solution for the illustrative model.

\subsection{Remarks on the Need for the Integral Equations (3)}

Suppose $D x(s)$ in (3) is a continuous function of $s$ over $s \geq 0$. Then, using the fact that equation (3) holds for all $t \geq 0$, it follows by the Fundamental Theorem of the Integral Calculus (found in any good calculus textbook) that

$$
D x(s)=\frac{\partial x(s)}{\partial t}, \quad s \geq 0
$$

where $\partial x(s) / \partial t$ denotes the derivative ${ }^{7}$ of the state vector $x$ with respect to time, evaluated at the particular time point $s$. Consequently, equations (3) and (5) fully determine the motion of the state vector $x(t)$ over times $t \geq 0$, conditional on the exogenous variables $\alpha(t)$ for $t \geq 0$, the exogenous function $f(\cdot)$, and the exogenously given initial state vector $x(0)$.

On the other hand, the crucial equation (6) expressing $D x$ as the time-derivative of $x$ does not follow from model equations (1) and (2) alone. In particular, simply labeling a vector as " $D x(t)$ " in model equation (2) does not guarantee that this vector is indeed the time- $t$ derivative of the state vector $x(t)$ in model equation (1). The relationship between $D x(t)$ and $x(t)$ at each time $t$ is entirely determined by the model structure. If the equations

\footnotetext{
${ }^{7}$ For $s=0$, only the right derivative of $x$ is well-defined. See Section 4.5 for further discussion of this point.
} 
(3) are not included among the model equations, there is no reason to expect that $D x(t)$ will be the time-derivative of $x(t)$ at any time $t \geq 0$.

Many theoretical studies of continuous-time state-space models do not explicitly include among the model equations the integral constraints (3) needed to ensure that $D x$ is indeed the time-derivative of $x$; these integral equations are implicitly assumed without comment. However, if a computer is being used to obtain an approximate numerical solution for the model, then all of the model's constraints (in appropriate approximate form) must be explicitly imposed on these variables. A computer will not automatically impose the integral constraints (3) that ensure $D x$ is the time-derivative of $x$ if these integral constraints (in some appropriate approximate form) are not included in the coding.

\subsection{Remarks on the Differentiability of the State Vector}

For many continuous-time state-space models in equation form, it cannot be assured that solution values are continuous functions of time. In particular, for the illustrative model set out in Section 4.1, it cannot be assured that the solution values for $z(t)$, and $D x(t)$ are continuous functions of $t$. This follows because the continuity of $z(t)$ and $D x(t)$ would imply that these vectors, meant to be time- $t$ endogenous, were in fact predetermined by their past realizations $z(\tau)$ and $D x(\tau)$ for $\tau<t$. In the latter case, the time- $t$ model equations would be over-determined in the sense that there would be no degree of freedom left at time $t$ to ensure that these equations could be satisfied.

Suppose $z(t)$ and $D x(t)$ are only right continuous, meaning that $z(q)$ and $D x(q)$ are only guaranteed to converge to $z(t)$ and $D x(t)$, respectively, as long as $q \rightarrow t$ along a path for which $q>t$. This implies, in particular, that $D x(s)$ in (3) can jump discontinuously at some points $s$ as long as it satisfies

$$
\lim _{q \rightarrow s, q>s} D x(q)=D x(s) .
$$

In this case, equation (6) must be weakened to

$$
D x(s)=\left.\frac{\partial x(s)}{\partial t}\right|_{+}=\lim _{q \rightarrow s, q>s}\left[\frac{x(q)-x(s)}{q-s}\right], \quad s \geq 0,
$$


where the right-hand term in (8) defines the right time-derivative of $x$, evaluated at $s$. Intuitively, this means that, at each time $s \geq 0, x(s)$ has a derivative approaching $s$ "from the right" (i.e., from times $q>s$ ). However, at some times $s^{\prime}$ it could happen that $x\left(s^{\prime}\right)$ has a kink point ("sharp corner") or a discontinuity, implying that $x$ is not differentiable at $s^{\prime}$.

In the remainder of these notes it is assumed for simplicity of exposition that the ordinary time-derivative $\partial x(s) / \partial t$ exists at each time $s \geq 0$.

\section{$5 \quad$ Discrete-Time State-Space Modeling in Equation Form}

Consider the illustrative continuous-time state-space model presented in Section 4.1. This model can be converted into discrete-time approximate form by replacing the derivative expressions $D x(t)$ with suitable discrete-time approximations.

For example, suppose $t$ is any given non-negative time point, and let $\Delta$ denote a positive time increment whose length is measured in some given time unit (e.g., in hours). Let the derivative $D x(t)$ at time $t$ be approximated by the following simple finite-difference expression:

$$
D x(t) \approx \frac{x(t+\Delta)-x(t)}{\Delta}
$$

Substituting (9) in place of $D x(t)$ in (2), and manipulating terms, one obtains

$$
x(t+\Delta) \approx S(z(t), x(t), \alpha(t)) \cdot \Delta+x(t)
$$

Note the important appearance of the time increment $\Delta$ on the right-hand side of equations (10). Even if $\Delta$ is set to 1 unit of time (e.g., 1h), it cannot be omitted from this equation. The expressions $x(t+\Delta)$ and $x(t)$ are measured in $x$ units; however, $S(z(t), x(t), \alpha(t))$ is

measured in $x$ per $t$, and it must be multiplied by $\Delta$ in order to obtain a commensurable expression in $x$ units.

Now let $t^{o}$ denote any given initial starting point along the real time line $[0, \infty)$, and let $\Delta$ denote a time increment whose length is expressed in some given unit of time (e.g., in hours). 
For each $j=0,1,2, \cdots$, define period $j$ to be the half-open time interval $\left[t^{o}+j \Delta, t^{o}+(j+1) \Delta\right)$. Finally, define

$$
F\left(z_{j}, x_{j}, \alpha_{j}\right) \equiv S\left(z_{j}, x_{j}, \alpha_{j}\right) \cdot \Delta+x_{j}
$$

where, for each $j=0,1,2, \cdots$,

$$
\begin{array}{r}
z_{j}=z\left(t^{o}+j \Delta\right) \\
x_{j}=x\left(t^{o}+j \Delta\right) \\
\alpha_{j}=\alpha\left(t^{o}+j \Delta\right)
\end{array}
$$

Then the original continuous-time state-space model over times $t \geq t^{o}$ can be expressed in discrete-time approximate form over periods $j \geq 0$, as follows:

Discrete-time approximation equations for periods $j \geq 0$ :

$$
\begin{array}{rrr}
\text { Simultaneous: } & 0=H\left(z_{j}, x_{j}, \alpha_{j}\right) \\
\text { Dynamic: } & x_{j+1}=F\left(z_{j}, x_{j}, \alpha_{j}\right)
\end{array}
$$

\section{Variables and functions:}

$$
\begin{aligned}
& x_{j}=\left(x_{j, 1}, \ldots, x_{j, n}\right) \in R^{n}, \quad j=0,1,2, \cdots \\
& \alpha_{j}=\left(\alpha_{j, 1}, \ldots, \alpha_{j, k}\right) \in R^{k}, \quad j=0,1,2, \cdots \\
& H: R^{m+n+k} \rightarrow R^{m} \text { and } F: R^{m+n+k} \rightarrow R^{n}
\end{aligned}
$$

\section{Classification of variables and functions:}

Period-j endogenous variables for periods $j \geq 0: z_{j}, x_{j+1}$

Period-j predetermined (state) variables for periods $j>0: x_{j}$

Exogenous variables: $x_{0}$, and $\alpha_{j}$ for periods $j \geq 0$ 
Exogenous functions: $H: R^{m+n+k} \rightarrow R^{m}$ and $F: R^{m+n+k} \rightarrow R^{n}$

It is important to note that, by construction, this discrete-time approximation converges to the original continuous-time state-space model over the time interval $\left[t^{o}, \infty\right)$ as the periodlength $\Delta$ converges to 0 . This is an important check on the basic logical consistency of the discrete-time approximation.

\section{Basic Causal Systems}

If a state-space model in equation form has a closed-form solution, the modeler has a way to greatly facilitate the study of its dynamic properties.

To see this, suppose a state-space model in equation form has a closed-form solution over some time interval $T$. Let all of the model's endogenous variables apart from state variables be substituted out as functions of the state variables and exogenous model elements. Let the resulting reduced-form model be called the Basic Causal System (BCS) for the original model. The modeler can use this BCS to explore the dynamic properties of the solution state vector. Once this is done, the modeler can derive dynamic properties for the solution values for the other endogenous variables using their earlier obtained expressions as functions of the state variables.

To illustrate the derivation of a BCS, consider once again the illustrative continuous-time state-space model in equation form that was set out in Section 4.1. Using the definition of a BCS, and the model solution developments in Section 4.3, it follows that the BCS for this model is as given below:

\section{BCS model equations for times $t \geq 0$ :}

$$
\begin{aligned}
D x(t) & =f(x(t), \alpha(t)) \\
x(t) & =\int_{0}^{t} D x(s) d s+x(0)
\end{aligned}
$$

\section{BCS variables and functions:}

$$
D x(t)=\left(D x_{1}(t), \ldots, D x_{n}(t)\right) \in R^{n}, t \geq 0
$$




$$
\begin{aligned}
& x(t)=\left(x_{1}(t), \ldots, x_{n}(t)\right) \in R^{n}, t \geq 0 \\
& \alpha(t)=\left(\alpha_{1}(t), \ldots, \alpha_{k}(t)\right) \in R^{k}, t \geq 0 \\
& f: R^{n+k} \rightarrow R^{n}
\end{aligned}
$$

\section{BCS classification of variables and functions:}

Time-t endogenous variables for $t \geq 0: D x(t)$

Time-t predetermined (state) variables for $t>0: x(t)$

Exogenous variables: $x(0)$, and $\alpha(t)$ for $t \geq 0$

Exogenous function: $f: R^{n+k} \rightarrow R^{n}$

This BCS can in principle be solved by a variety of techniques. Let

$$
\boldsymbol{x}=(x(t))_{t \geq 0} \text { and } \boldsymbol{\alpha}=(\alpha(t))_{t \geq 0} .
$$

Then the solution to the BCS takes the general form

$$
\boldsymbol{x}=v(\boldsymbol{\alpha}, x(0), f(\cdot)) .
$$

\section{Presentation Principles for Economic State-Space Models in Equation Form}

Six recommended steps for the presentation of economic state-space models in equation form are as follows:

Step 1: Provide a complete and consistent set of model equations. By complete is meant that the model equations provide enough information to permit (in principle) the determination of a solution value for each endogenous variable. By consistent is meant that the model equations do not contradict each other. 
Step 2: Provide a classification of variables and functions. Partition the model variables and functions into time-t endogenous, time-t predetermined, and exogenous for each time point (or period) $t$.

Step 3: Explain the intended economic meaning of each model variable and function. For example, $K(t)$ denotes the time- $t$ capital stock, $F(K, L)$ denotes an aggregate production function, and so forth.

Step 4: Specify admissibility restrictions on exogenous variables and functions. Admissibility restrictions are conditions imposed on exogenous variables and functions in order to guarantee the economic plausibility of the model structure and model solutions. An example would be conditions imposed on exogenous variables and functions in order to ensure the non-negativity of an endogenously determined capital stock $K(t)$ at each time $t$.

Step 5: Provide an economic interpretation for the model equations, both individually and as a whole. In particular, explain for each model equation whether it is an accounting identity, an assumed type of behavior for an economic decision maker, an imposed market equilibrium condition, or some other form of relationship. Also, what kind of economic process is the model as a whole meant to represent?

Step 6: Provide a complete description of any modeled optimization problem. In particular, explain what entity is undertaking the optimization, and provide a complete and careful description of the objective function, the decision variables, the feasible decision sets, and the constraints for this optimization problem. Also explain how this optimization problem is to be represented in state-space form (e.g., by means of its binding first-order necessary conditions).

\section{Analysis of Economic State-Space Models in Equation Form}

Below are six issues that have traditionally been studied for economic state-space models in equation form. 


\section{Issue 1: Existence of Solutions}

(a) A state-space model consisting of a system of equations together with a classification of variables and functions is said to have a solution if values can be found for the model's endogenous variables that satisfy the following two criteria: (i) These values depend only on the model's exogenous variables and functions; and (ii) these values satisfy the system of equations, conditional on a particular specification of values for the model's exogenous variables and a particular specification of forms for the model's exogenous functions. Do solutions exist?

(b) A solution expressible in terms of exogenous variables and functions that satisfy all modeler-specified admissibility restrictions is referred to as an admissible solution. Do admissible solutions exist?

\section{Issue 2: Equilibrium and Coordination Aspects of Admissible Solutions}

(a) Do markets clear?

(b) Are plans realized?

(c) Are expectations fulfilled?

(d) Are other types of equilibrium properties in evidence, e.g., are admissible solutions necessarily Nash equilibria?

(e) Do admissible solutions display coordination failure, i.e., are they Pareto-dominated Nash equilibria?

\section{Issue 3: Dynamic Properties of Admissible Solutions}

(a) Does the system have a BCS? ${ }^{8}$

\footnotetext{
${ }^{8}$ In the older economic growth literature, a dynamic model represented in the form of a system of ordinary differential equations is said to be non-causal if there exists at least one reachable point $x(t)$ at which $D x(t)$ is not uniquely determined. For non-causal models, the introduction of some form of external selection principle would be needed in order to resolve the ambiguity in $D x(t)$ and hence in the continuation of the solution path. It follows that non-causal dynamic models do not have a BCS. For example, Uzawa's classic continuous-time two-sector growth model [9] is known to be non-causal under some parameter specifications.
} 
(b) If the answer is yes, derive or characterize the admissible solutions for this BCS.

(c) Use this derivation or characterization to infer the dynamic properties of the state vector, e.g., by means of phase diagram techniques.

(d) Use the dynamic properties of the state vector to derive dynamic properties for all other endogenous variables of interest.

\section{Issue 5: Stability Properties of Equilibrium Admissible Solutions}

(a) For any given definition of an equilibrium, which (if any) equilibrium admissible solutions exhibit local (in)stability?

(b) For any given definition of an equilibrium, which (if any) equilibrium admissible solutions exhibit global (in)stability?

(c) For any given definition of an equilibrium, which (if any) equilibrium admissible solutions take the form of (un)stable limit cycles?

\section{Issue 6: Optimality Properties of Admissible Solutions}

(a) Are admissible solutions efficient, in the sense that no physical resources are wasted?

(b) Are admissible solutions Pareto-efficient, in the sense that there is no wastage of opportunity to improve the welfare of any one modeled human agent in a way that does not diminish the welfare of any other modeled human agent?

(c) Are admissible solutions socially optimal in the sense of maximizing some meaningful measure of social welfare? 


\section{Agent-Based Economic Modeling: Introduction}

Agent-Based Computational Economics (ACE) is the computational modeling of economic processes (including whole economies) as open-ended dynamic systems of interacting agents. ${ }^{9}$ Here agent refers broadly to a bundle of data and methods representing an entity constituting part of the system.

The agents comprising an ACE model often include an overarching "world" agent whose data determine the physical realm (e.g., spatial landscape) within which the remaining agents interact. The world-agent's methods typically provide simulation controls accessible to the modeler, such as simulation stopping rules and non-perturbational instrumentation devices (e.g., pause-to-observe buttons).

Other types of agents appearing in ACE models include: individuals (e.g., consumers, workers, and managers); social groupings (e.g., families, firms, communities, and government agencies); institutions (e.g., credit, market, and regulatory systems); biological entities (e.g., crops, livestock, and forests); and physical entities (e.g., buildings, highways, weather, and geographical regions). The initial data of these agents determine their fixed attributes (e.g., learning capabilities), initial settings for their variable attributes (e.g., available resources), and seed values for any pseudo-random number generators available to them. The initial methods of these agents determine how they initially interact within their world, and to what extent they can change their methods over time based on the outcomes of these interactions.

In short, agents in ACE models can range from passive system features to active datagathering decision-makers (DMAgents) capable of sophisticated learning and social behaviors. Moreover, agents can be composed of other agents, which permits the study of hierarchical aspects of economic systems; see Fig. 1.

ACE models are discrete-time state-space models. An ACE modeling of a dynamic system is typically implemented as a software program that determines the motion of the system

\footnotetext{
${ }^{9}$ Annotated pointers to ACE tutorials, publications, demos, software, research groups, and research area sites are posted at the ACE website [10]. Some of the materials in this section are adapted from the introduction at this website.
} 


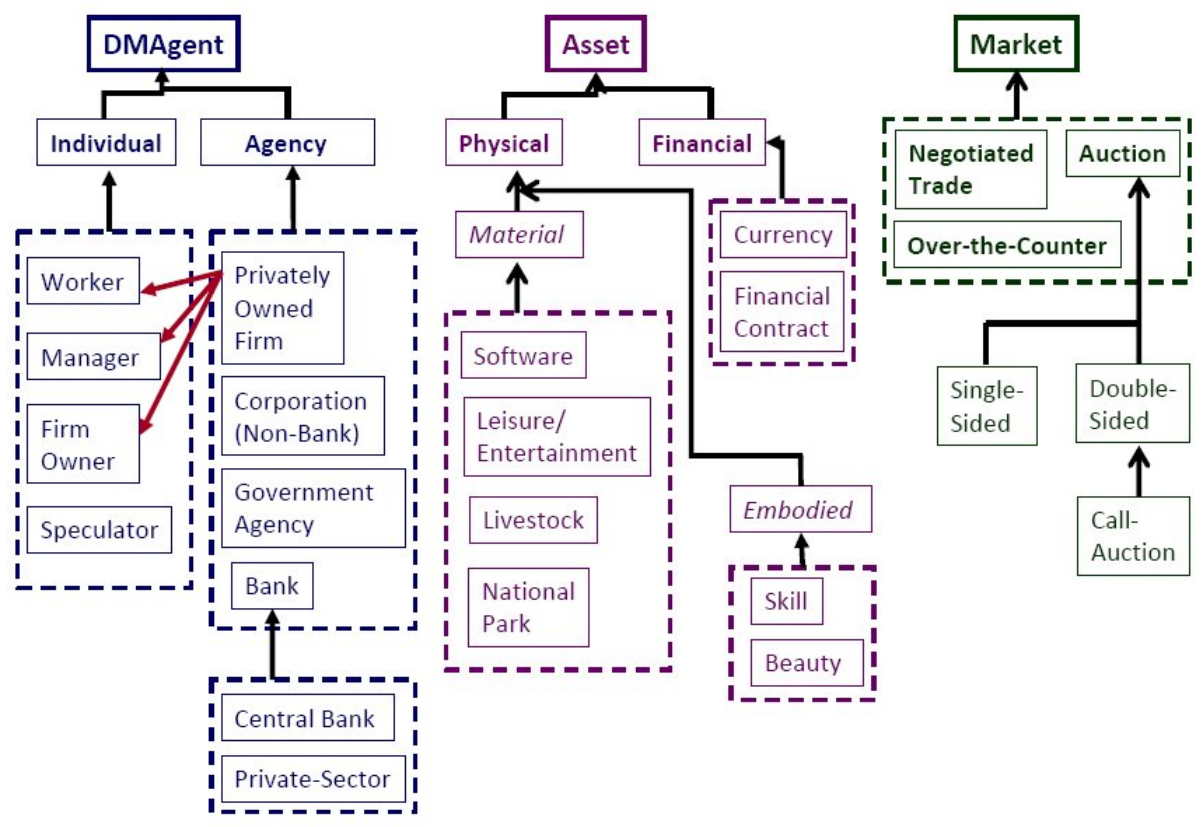

Figure 1: Partial hierarchy of agents for a decentralized market economy. Upward-pointing (black) arrows denote "is a" relationships and downward-pointing (red) arrows denote "has a" relationships. Source: [11]

state over successive time periods, starting from a user-specified initial system state. The system state at the beginning of each time period consists of the internal data and methods of each constituent agent. After the configuration of the initial state by the modeler, the system state is permitted to change over time driven solely by agent interactions. The only further role permitted for the modeler in this dynamic process is the non-perturbational observation and recording of model outcomes of interest.

In principle, an ACE model can be expressed analytically as a discrete-time system of coupled recursive functions; but this representation would be extremely complicated. Instead, ACE models are more naturally explained in terms of verbal descriptions, flow diagrams, and/or pseudo-code expressing the logical flow of agent processes and interactions over time, as an accompaniment to the actual underlying code.

For example, Fig. 2 depicts the flow of events in an ACE modeling of a goods market organized as a double auction (DA). The participants in this DA are profit-seeking traders 


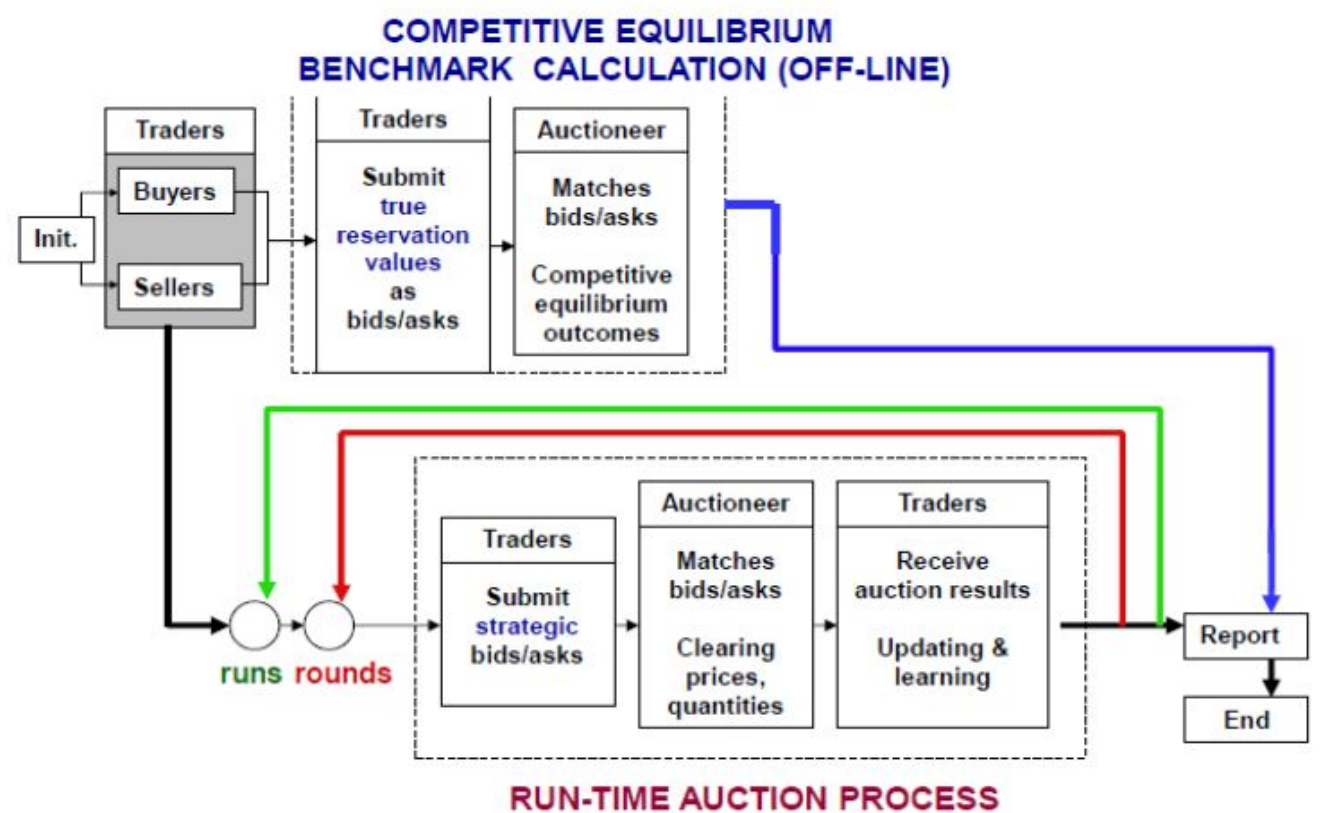

Figure 2: Flow diagram for an ACE double-auction market study.

who submit bids and asks for goods in successive trading rounds. Each bid takes the form of a descending schedule depicting a buyer's willingness to pay for each successive unit of good, and each ask takes the form of an ascending schedule depicting a seller's minimum acceptable sales price for each successive unit of good. The DA matches bids to asks for all inframarginal units at which bids are at least as great as asks, and determines the resulting market clearing price as the midpoint between the marginal bid and ask. Buyer-seller trades then take place at this market clearing price, resulting in a (possibly zero) profit outcome for each trader.

An interesting aspect of this DA modeling is that the traders have learning capabilities. At the end of each trading round, each trader uses its profit outcome to update its bid or ask for the next trading round. Thus, bids and asks can deviate from true reservation values.

Figure 2 outlines one possible sensitivity design of interest for this DA modeling. Multiple runs of this DA market could be conducted under systematically varied trader learning methods, where each run consist of a specified number of trading rounds. The resulting strategic DA profit outcomes could then be compared with "competitive equilibrium" DA 
profit outcomes in which submitted bids and asks are required to represent true buyer and seller reservation values. These comparisons could be used to explore which learning methods (if any) permit the buyers or sellers to exercise strategic market power, i.e., to implement learned bid/ask behaviors resulting in sustained profit advantages relative to the profit outcomes they would achieve under competitive equilibrium.

More generally, market conditions (e.g., relative number of buyers versus sellers), DA market rules (e.g., pricing mechanisms and restrictions on bid/ask forms), and other trader attributes (e.g., specifications for true reservation values) could also be systematically varied. This would permit a fuller understanding of both strategic and structural market power advantages in DA contexts. For such an ACE DA study, see [12].

The ACE DA study depicted in Fig. 2 is an example of ACE research directed towards a normative objective: namely, good market design. More broadly, ACE researchers pursuing normative understanding are interested in evaluating whether designs proposed for economic policies, institutions, or processes will result in socially desirable system performance over time. Examples of this form of ACE research can be found at resource sites [13] through [15].

The general approach taken by ACE researchers pursuing normative design studies is akin to filling a bucket with water to determine if it leaks. A computational world is constructed that captures the salient aspects of an economic system operating under a specified design. The world is then populated with decision-making agents with learning capabilities and allowed to develop over time. One key issue is the extent to which the resulting world outcomes are efficient, fair, and orderly, despite attempts by the decision-making agents to gain individual advantage through strategic behavior. A second key issue is a cautionary concern for adverse unintended consequences.

A second primary objective of ACE research is empirical understanding: Why have particular observed regularities evolved and persisted despite the absence of top-down planning and control? Examples of such regularities include trade networks, socially accepted monies, market protocols, business cycles, and the common adoption of technological innovations.

ACE researchers pursuing this second objective seek explanations for empirical regu- 
larities grounded in the repeated interactions of agents operating in realistically rendered computational worlds. Specifically, they try to understand whether particular types of observed regularities can be reliably generated within these worlds. A particularly active ACE research area along these lines is agent-based financial economics; see [16].

A third primary objective is qualitative insight and theory generation: How can ACE models be used to gain a better understanding of dynamic economic systems through a better understanding of their full range of potential behaviors over time (equilibria plus basins of attraction)? Such understanding would help to clarify not only why certain types of regularities have evolved and persisted but also why others have not.

A quintessential example of this third objective is the desire to resolve a long-standing issue first raised by economists such as Adam Smith (1723-1790), Ludwig von Mises (18811973), and Friedrich von Hayek (1899-1992): namely, what are the self-organizing capabilities of decentralized market economies? As evidenced by the extensive materials posted at the resource site [17], this is a particularly active ACE research area.

A fourth primary objective is methodological advancement: How best to provide ACE researchers with the methods and tools they need to undertake theoretical studies of dynamic economic systems through systematic computational sensitivity tests, and to examine the compatibility of sensitivity-generated theories with real-world data? As documented at the resource site [18], ACE researchers are exploring a variety of ways to address this fourth objective, ranging from careful considerations of methodological principles to the practical development of programming, visualization, and empirical validation tools. An interesting recent development, surveyed in [19], is a focus on the development of a common standard for agent-based modeling descriptions.

\section{ACE Presentation and Analysis: General Principles}

In studies relying on ACE modeling, researchers typically carry out the presentation and analysis of their models by means of the following eight steps: ${ }^{10}$

\footnotetext{
${ }^{10}$ These steps are adapted from [11].
} 
Step One: Computationally construct an ACE model consisting of a collection of agents tailored to the study of a theoretical issue of interest.

Step Two: Develop a sensitivity design suitable for the systematic exploration of this theoretical issue.

Step Three: Configure the ACE model in accordance with the sensitivity design.

Step Four: Compile and run the ACE model with no further modeler interference, and record model outcomes of interest.

Step Five: If the ACE model includes stochastic elements, ${ }^{11}$ repeat each sensitivity test multiple times for multiple stochastic element realizations to generate an ensemble of runs from which sample distributions for recorded modeloutcomes can be derived.

Step Six: Repeatedly iterate steps three through five until the full range of configurations specified under the sensitivity design has been explored.

Step Seven: Analyze the resulting sample distributions for recorded model outcomes and summarize their theoretical implications.

Step Eight: Use these theoretical summaries to form hypotheses (conjectures) that can be brought to historical or real-time data for testing and empirical validation.

\section{Concluding Remarks}

These introductory notes have covered general presentation and analysis principles for economic state-space models, whether in equation or agent-based form. The primary intended readership is graduate students of economics, early in their careers, who plan to support their thesis research by some form of dynamic modeling effort.

\footnotetext{
${ }^{11}$ These stochastic elements can take the form of output from pseudo-random number generators (e.g., "coin flips" to resolve indifference among available choices) or "truly" random data (e.g., weather events) streamed into the ACE model from the real world.
} 
An attempt has been made to keep the exposition concise and to the point, to maintain readability. However, suggestions regarding additional relevant reference materials would be most welcome.

\section{Acknowledgement}

I am grateful to my Ph.D. economics students Jiaqi Ge, Deung-Yong Heo, Dong-Jin Pyo, and Kate Sinitskaya, and to the many M.S. economics students who have taken my Econ 502 course [1], for helpful discussions on the topics addressed in these notes.

\section{References}

[1] Leigh Tesfatsion, Econ 502 Syllabus: M.S.-Level Macroeconomic Theory, http: //www.econ.iastate.edu/classes/econ502/tesfatsion/sy1502.htm

[2] Leigh Tesfatsion, Experimental Design: Basic Concepts and Terminology, http: //www.econ.iastate.edu/tesfatsi/expdesign.pdf

[3] Leigh Tesfatsion, The Basic Solow-Swan Descriptive Growth Model, http://www.econ.iastate.edu/classes/econ502/tesfatsion/solowmod.pdf

[4] Leigh Tesfatsion, A Simple Illustrative Optimal Growth Model, http://www.econ.iastate.edu/classes/econ502/tesfatsion/OptGrow.pdf

[5] Akira Takayama, 1985, Mathematical Economics, 2nd Ed., Cambridge Univ. Press, UK.

[6] Russell Cooper, Dynamic Programming: An Overview, February 14, 2001, http://www.econ.iastate.edu/tesfatsi/dpintro.cooper.pdf

[7] Gianluca Violante, Notes on Discrete Time Stochastic Dynamic Programming, Spring 2000, http://www.econ.iastate.edu/tesfatsi/dpbasic.violante.pdf

[8] Leigh Tesfatsion, Notes on the Lucas Critique, Time Inconsistency and Related Issues, http://www.econ.iastate.edu/tesfatsi/luccrit.pdf 
[9] Hirofumi Uzawa, "On a Two-Sector Model of Economic Growth, II," Review of Economic Studies, Vol. 19, pp. 105-118.

[10] Leigh Tesfatsion, Agent-Based Computational Economics: Growing Economies from the Bottom Up, http: //www.econ.iastate.edu/tesfatsi/ace.htm

[11] Paul L. Borrill and Leigh Tesfatsion, 2011, "Agent-Based Modeling: The Right Mathematics for the Social Sciences?," pp. 228-258 in J.B. Davis and D.W. Hands (eds.), Elgar Companion to Recent Economic Methodology, Edward Elgar Publishers, 560pp. http://www. econ.iastate.edu/tesfatsi/ABMRightMath.PBLTWP.pdf

[12] James Nicolaisen, Valentin Petrov, and Leigh Tesfatsion, 2001, "Market Power and Efficiency in a Computational Electricity Market with Discriminatory Double-Auction Pricing," IEEE Transactions on Evolutionary Computation, Vol. 5, No. 5, October, pp. 504-523. http://www.econ.iastate.edu/tesfatsi/mpeieee.pdf

[13] Leigh Tesfatsion, ACE Research Area: Economic Policy Analysis, http://www.econ.iastate.edu/tesfatsi/apolicy.htm

[14] Leigh Tesfatsion, ACE Research Area: Agricultural and Natural Resource Issues, http://www. econ.iastate.edu/tesfatsi/aagric.htm

[15] Leigh Tesfatsion, Agent-Based Electricity Market Research, http://www.econ.iastate.edu/tesfatsi/aelect.htm

[16] Leigh Tesfatsion, Agent-Based Financial Economics, http: //www.econ.iastate.edu/tesfatsi/afinance.htm

[17] Leigh Tesfatsion, Agent-Based Macroeconomics, http://www.econ.iastate.edu/tesfatsi/amulmark.htm

[18] Leigh Tesfatsion, Verification and Empirical Validation of Agent-Based Computational Models, http://www.econ.iastate.edu/tesfatsi/empvalid.htm 
[19] Birgit Müller et al., 2014, "Standardised and Transparent Model Descriptions for AgentBased Models: Current Status and Prospects," Environmental Modelling \& Software, Vol. 55, pp. 156-163.

http://www.sciencedirect.com/science/article/pii/S1364815214000395 